\title{
Implementation of In-Home Activity Recognition Based on Web Service and Machine Learning Approaches
}

\author{
Wei-Hao Chen, Ta-Chou Chiu, Wen-Hui Chen* \\ Graduate Institute of Automation Technology, \\ National Taipei University of Technology \\ Taipei, Taiwan \\ *Corresponding Author: whchen@ ntut.edu.tw
}

\begin{abstract}
Human Activity recognition systems need sensor deployment to acquire activity information. How to make good use of smartphones as mobile sensing devices to develop a real-time recognition system for context-aware applications has become an active research topic in recent years. In this study, we implemented a mobile activity monitoring system and compared the performance of some well-known machine learning approaches when sensor data collected from practical smartphone users is limited. The evaluation is based on the records of accelerometer and gyroscope measurements collected from six different activities of daily living, including sitting, lying, standing, walking, going upstairs, and going downstairs.
\end{abstract}

Keywords: smart homes, activity recognition, mobile device sensors, machine learning.

\section{Introduction}

According to a report that indicates people aged 60 or more account for $12 \%$ of the current global population, and that number is estimated to rise to $22 \%$ by 2050 [1]. The rising aging population has impacts on both the economy and social organization of a country. Most of the elders tend to live in their homes independently instead of living in a nursing home. Therefore, it is important to find alternatives for senior care with the assistance of information technology to cope with the fast-aging population.

To maintain the independence of elders, many intelligent assistive systems (IAS) by employing various sensors have been proposed in recent years. The authors in [2] presented an intelligent wheelchair to enable mobility of cognitively impaired elders. In [3], the authors adopted pervasive computing to help people to perform multi-step tasks, such as making a cup of tea or coffee. In [4], the authors reported the use of a wearable camera with an automatic recording function for lifelogging and showed how this device can be employed to store and access information of our daily life.

Human activity recognition is an active research topic and the key step required for building the IAS to many potential applications. Numerous approaches to activity recognition for various applications have been proposed in past research [5]-[8]. The demand for understanding human activities in healthcare applications has increased. With the advancement of information and communication technologies (ICT), the development of ICT-based assistive systems is considered as a potential solution to help the elderly live independently.

Nowadays, smart handheld devices such as smartphones and tablets have become popular and affordable. Modern smartphones have equipped with inertial sensors such as accelerometers, gyroscopes, and digital compasses. These mobile devices in our daily lives provide us with rich information in a real-time fashion. By taking good use of unobtrusive smartphone sensors, an assistive system can be built for monitoring the activities of elders and making further analysis through the use of information technology for improving the quality of daily living.

A number of activity recognition studies using smartphones have been conducted over the past few decades [9]-[12]. Most of the studies in sensor-based activity recognition relied on hand-crafted features. In recent years, the development of deep learning has achieved great success 
in various areas and has become a trending research direction in activity recognition [13]-[14]. Deep learning requires sufficient data to train network parameters, which is time-consuming and needs labor-intensive data labeling. When the training data is limited, the performance of deep learning model is not quarantined performing well. Therefore, the majority of the previous works are done with sufficient dataset and claimed that deep learning outperforms many state-of-the-art approaches. However, not every task can easily provide sufficient data for deep learning in practice.

In this paper, in addition to implementing a real-time activity recognition system based on smartphone sensors, we also examine and compare the performance of some popular learning models when the dataset is not large enough. The remainder of this paper is organized as follows. In the next section, we introduce the proposed client-server system that realizes real-time activity recognition based on smartphone sensors and discuss the data collection setup for the experiment. Experimental results and a comparison of recognition accuracy of four different learning algorithms are presented in Section III and finally, Section IV concludes this paper, including future work.

\section{System Description}

In this section, we describe the framework of the proposed system and the process of sensor data collection for experiments.

\subsection{System Architecture}

Although the constant development of smartphones makes them became technological mainstays of computing devices in our everyday life, mobile devices still face limitations of computation resources compared to desktop computers. As our focus was on monitoring activities on mobile devices, we have to deal with the issue of computational limitation. Therefore, we adopted a client-server architecture and established a remote server for sensor data processing and the development of recognition algorithm, as shown in Fig.1.

As can be seen from Fig. 1, the framework of the proposed system partitions tasks into the client side and the server side through the web service. In the client side, the mobile device sends the sensor signal to the remote web service server over the HTTP protocol. The remote server is responsible for sensor data processing and storage on an
SQL database implemented with Microsoft SQL Server, and activity inference over the Internet. The remote server also provides a web-based interface that can be used for data storage and inquiry through a mobile app.

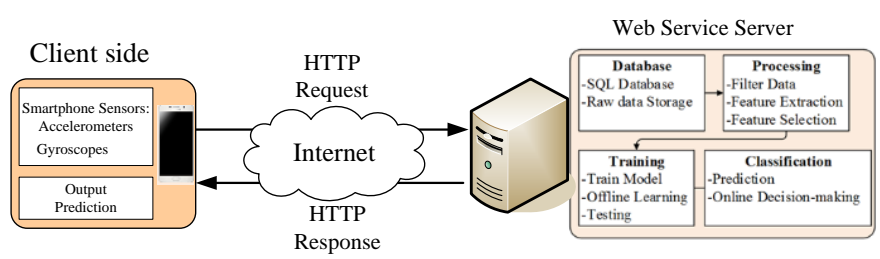

Fig. 1 Architecture of the proposed client-server system for sensor data collecton

\subsection{Data Collection Setup}

In order to collect sensor data for training the recognition model, we designed a data collection app on a smartphone. The flowchart of the mobile app for data collection is shown in Fig. 2. When executing, the app program starts initialization and waits for the user to select an ID and the activity (denoted as activity ID) to be performed. When the START button is pressed, the data gathered from built-in accelerometers and gyroscopes of a smartphone will be transmitted to the remote server at a refresh rate of $20 \mathrm{~ms}$. The design process aims to facilitate the data collection process and the annotation or data labeling for later use in supervised training. Fig. 3 shows a screenshot of sensor data collection with the designed app.

The self-collected dataset used in this experiment was built with a smartphone (Samsung Galaxy Note 5) from the recording of six participants performing six different activities of daily living, including walking, sitting, standing, lying, going upstairs, and going downstairs. A comparison of data variations captured by the 3 -axis accelerometer and 3-axis gyroscope embedded in the smartphone for six different activities was shown in Fig. 4. As can be seen from Fig. 4, the distinction of activities between dynamic and static activities (inter-class variation) is significant but less obvious among dynamic activities or static activities (intra-class variation). 


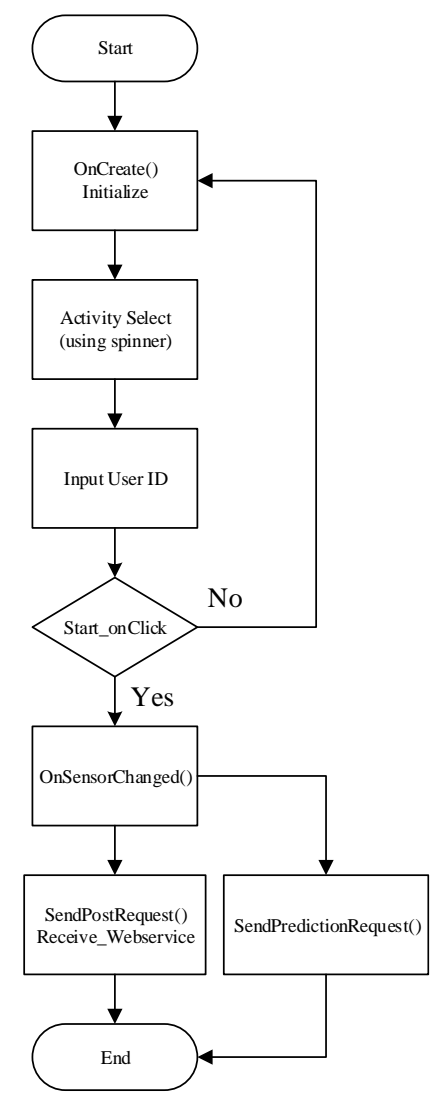

Fig. 2 The flowchart of the mobile app for data collection

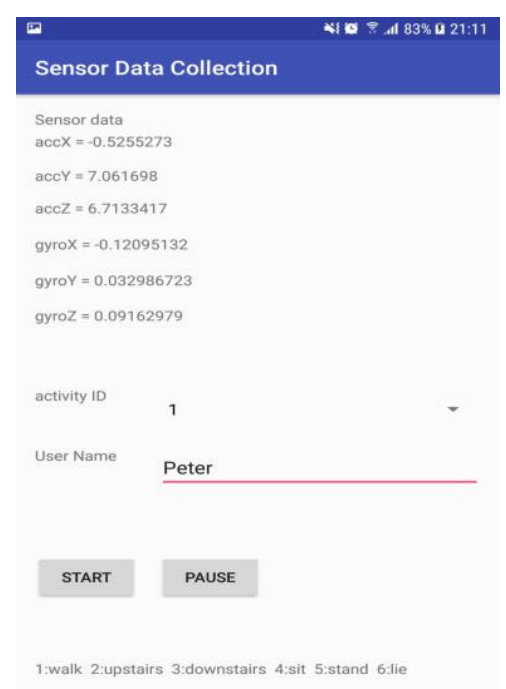

Fig. 3 A screenshot of sensor data collection with the designed app

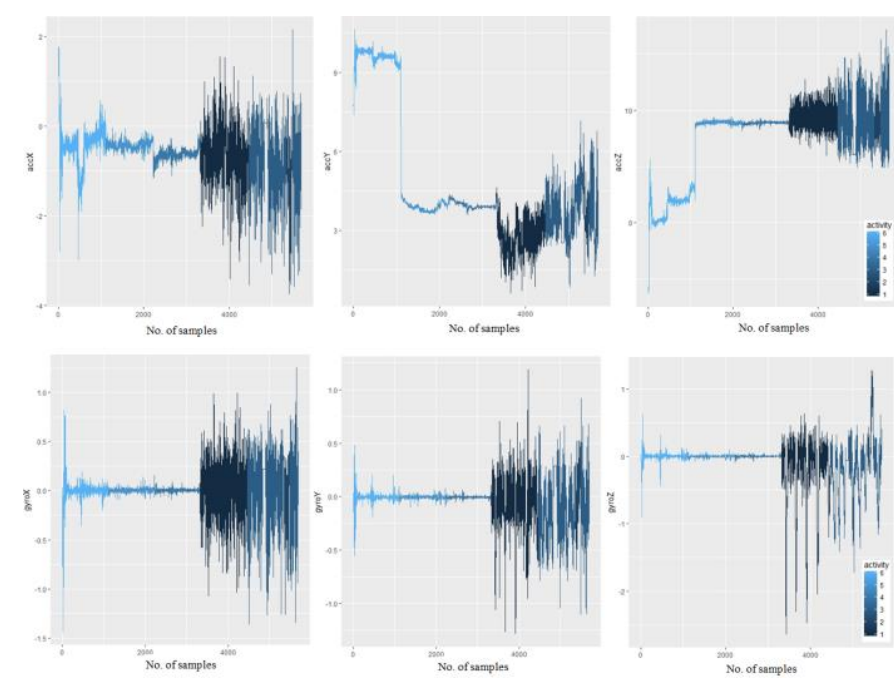

Fig. 4 Sensor data variations capturing from a 3-axis accelerometer (upper row) and a 3-axis gyroscope (bottom row) for six different activities.

\section{Experimental Results}

In this session, we evaluated the performance of recurrent neural networks (RNN) with long short-term memory (LSTM) cells and four high popularity learning algorithms to serve a comparison purpose using the self-collected dataset.

The goal of this experiment is to make a comparison of machine learning approaches when training data is limited. Therefore, we only took 12,353 records from the collected data as listed in Table 1. The dataset was randomly split into $70 \%$ and $30 \%$ for the training and the test stage, respectively.

Table 1. Data distribution of the experimental dataset

\begin{tabular}{|c|l|c|c|}
\hline ID & \multicolumn{1}{|c|}{ Activity } & $\begin{array}{c}\text { No. of } \\
\text { Records }\end{array}$ & Data Ratio \\
\hline 1 & Walking & 2,012 & $16.3 \%$ \\
\hline 2 & Going upstairs & 2,313 & $18.6 \%$ \\
\hline 3 & Going downstairs & 1,997 & $16.2 \%$ \\
\hline 4 & Sitting & 2,008 & $16.3 \%$ \\
\hline 5 & Standing & 2,013 & $16.3 \%$ \\
\hline 6 & Lying & 2,010 & $16.3 \%$ \\
\hline & Total & 12,353 & $100 \%$ \\
\hline
\end{tabular}

There are several performance metrics used in the study of classification tasks, such as confusion matrices, classification accuracy, precision, recall, and F-measure. We adopted the F-measure to evaluate the recognition 
performance. Defined in (1), the F-measure or F1-score is a measure of test accuracy by combining the measure that assesses the precision and recall scores in (2) and (3), respectively.

$$
\begin{gathered}
\text { F1 }- \text { score }=\frac{2 \times \text { Precision } \times \text { Recall }}{\text { Precision }+ \text { Recall }} \\
\text { Precision }=\frac{1}{N} \sum_{i=1}^{N} \frac{T P_{i}}{T P_{i}+F P_{i}} \\
\text { Recall }=\frac{1}{N} \sum_{i=1}^{N} \frac{T P_{i}}{T P_{i}+F N_{i}}
\end{gathered}
$$

where

$$
\begin{aligned}
& N \text { is the total number of class instances } \\
& T P \text { is true positive } \\
& F N \text { is false negative } \\
& F P \text { is false positive }
\end{aligned}
$$

We empirically examined the performance of four popular machine learning algorithms, namely decision trees, support vector machines, random forests, and naïve Bayes together with LSTM-RNN to evaluate which learning algorithm performed well when data is limited. A comparison of the model performance for each activity was listed in Table 2. We used $\boldsymbol{R}$ packages, C50, e1071, and randomForest with their default settings to implement the four machine learning models. As to the RNN model, we stacked two LSTM cells to form a deeper LSTM-RNN model using BasicLSTMCell and MultiRNNCell of Tensorflow to implement the LSTM-RNN model with the following parameter setting:

Time step: 5

Hidden number of feature: 32

Training iteration: 45,000

Batch size: 4,500

Learning rate: 0.0025

Lambda loss amount: 0.0015

Number of inputs: 6

Number of classes: 6

As can be observed, the LSTM-RNN model does not perform that well compared to popular machine learning models, such as random forests and support vector machines on a limited small dataset. This may result from the need of a large amount of data and fine-tune network

parameters for training deep neural networks to obtain good results. The performance of deep learning models is expected to increase as the scale of data increases. This is one of the most important differences between deep learning models and traditional machine learning models.

In this experiment, the best test accuracy goes to random forests. For the comparison purpose, the confusion matrices of random forests and LSTM-RNNs were listed in Table 3 and Table 4, respectively. The diagonal elements in the confusion matrix represent the number of activities for which the prediction is the same as the ground truth, while off-diagonal elements are those that are misclassified. Fig. 5 and Fig. 6 show a visual representation of the confusion matrix derived from the random forest and LSTM-RNN model, respectively.

Table 2. Accuracy comparisons for each activity compared among different learning models

\begin{tabular}{|l|l|c|c|c|c|}
\hline \multicolumn{1}{|c|}{ Methods } & $\begin{array}{l}\text { Decision } \\
\text { Trees } \\
\text { (C50) }\end{array}$ & $\begin{array}{c}\text { SVMs } \\
(\mathrm{e} 1071)\end{array}$ & $\begin{array}{c}\text { Random } \\
\text { Forests } \\
\text { (random } \\
\text { Forest) }\end{array}$ & $\begin{array}{c}\text { Naïve } \\
\text { Bayes } \\
(\mathrm{e} 1071)\end{array}$ & LSTM-RNNs \\
(Tensorflow)
\end{tabular}

Note: All accuracy scores were represented in percentages $(\%)$

Table 3 . The confusion matrix of random forests

\begin{tabular}{|l|c|c|c|c|c|c|}
\hline \multicolumn{1}{|c|}{ Activities } & W & U & D & SI & ST & L \\
\hline Walking (W) & $\mathbf{6 0 1}$ & 0 & 16 & 0 & 0 & 0 \\
\hline Upstairs (U) & 13 & 657 & 34 & 0 & 0 & 0 \\
\hline Downstairs (D) & 23 & 2 & 580 & 0 & 1 & 0 \\
\hline Sitting (SI) & 2 & 0 & 0 & 572 & 27 & 0 \\
\hline Standing (ST) & 2 & 0 & 0 & 14 & 568 & 0 \\
\hline Lying (L) & 0 & 0 & 0 & 0 & 0 & 594 \\
\hline
\end{tabular}


Table 4. The confusion matrix of LSTM-RNNs

\begin{tabular}{|l|c|c|c|c|c|c|}
\hline \multicolumn{1}{|c|}{ Activity } & W & U & D & SI & ST & L \\
\hline Walking (W) & $\mathbf{5 6 9}$ & 5 & 37 & 2 & 0 & 4 \\
\hline Upstairs (U) & 23 & $\mathbf{6 3 8}$ & 43 & 0 & 0 & 0 \\
\hline Downstairs (D) & 86 & 5 & $\mathbf{5 1 4}$ & 1 & 0 & 0 \\
\hline Sitting (SI) & 0 & 0 & 0 & $\mathbf{5 2 6}$ & 75 & 0 \\
\hline Standing (ST) & 3 & 0 & 0 & 41 & $\mathbf{5 4 0}$ & 0 \\
\hline Lying (L) & 0 & 0 & 2 & 0 & 0 & $\mathbf{5 9 2}$ \\
\hline
\end{tabular}

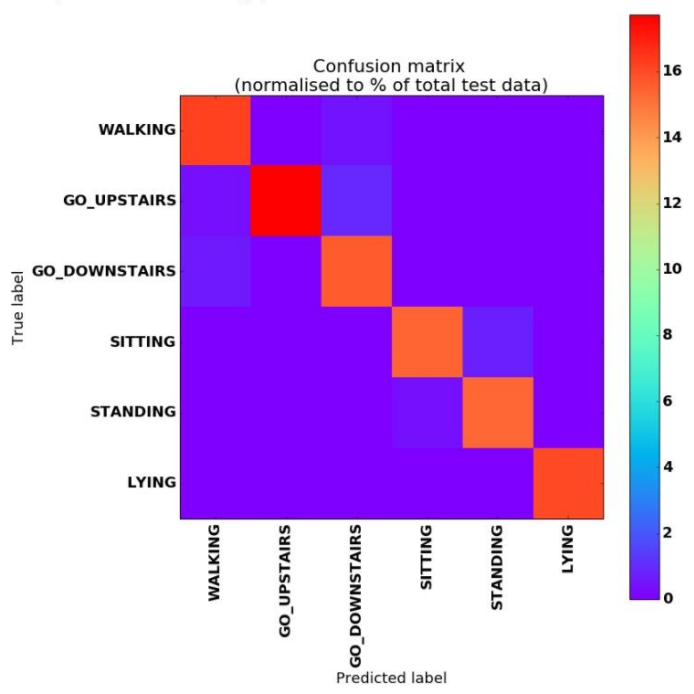

Fig. 5 A visual representation of confusion matrix derived from random forests

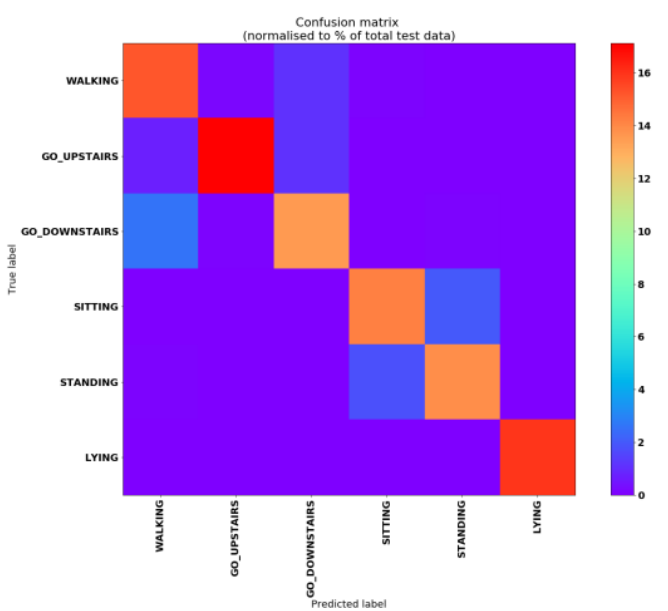

Fig. 6 A visual representation of confusion matrix derived from LSTM-RNNs

\section{Conclusions and Future Work}

The use of sensors embedded in smartphones is a convenient way for estimating different motion types, which can be used to identify in-home activities of daily living to achieve a real-time activity monitoring system. In this study, we have implemented an in-home activity recognition system based on the client-server architecture and investigated the recognition performance of LSTM-RNNs with four popular machine learning models with limited data.

Experimental results show that the random forests and support vector machines could outperform vanilla recurrent neural networks (RNNs) when data is insufficient. This indicated that the amount of data matters when it comes to model performance. As such, it is challenging to use deep learning models when the data is insufficient. We are currently conducting an ongoing experiment that uses generative models to augment data to resolve the small data problem based on deep learning research.

\section{Acknowledgment}

This work is supported by Taiwan Ministry of Science and Technology (MOST) under Grant No. MOST 106-2221-E-027-059.

\section{References}

(1) "Ageing in the Twenty-First Century: A celebration and a challenge," Published by the United Nations Population Fund (UNFPA), New York, 2012.

(2) A. Mihailidis, P. Elinas, J. Boger, and J. Hoey, “An intelligent powered wheelchair to enable mobility of cognitively impaired older adults: an anti-collision system," IEEE Trans. on Neural Systems and Rehabilitation Engineering, vol. 15, no. 1, March 2007.

(3) J. P. Wherton and A. F. Monk, "Problems people with dementia have with kitchen tasks: The challenge for pervasive computing," Interacting with Computers, vol. 22, no. 4, pp. 253-266, July 2010.

(4) S. Lindley, D. Randall, W. Sharrock, M. Glancy, N. Smyth and R. Harper, "Narrative, memory and practice: tensions and choices in the use of a digital artefact," In Proceedings of the 23rd British HCI Group Annual Conference on Human Computer Interaction, pp.1-9, 2009.

(5) J. K. Aggarwal and M. S. Ryoo, "Human activity analysis: a review," ACM Computing Surveys, vol. 43, no. 3, pp. 1-47, April 2011. 
(6) V. R. Jakkula and D. J. Cook, "Detecting anomalous sensor events in smart home data for enhancing the living experience," Proceedings of the 7th AAAI Conference on Artificial Intelligence and Smarter Living, pp. 33-37, 2011.

(7) W. H. Chen and W. S. Tseng, "A novel multi-agent framework for the design of home automation," Fourth International Conference on Information Technology, pp. 277-281, April 2007.

(8) W. H. Chen, Y. H. Lin, and S. J. Yang, "A generic framework for the design of visual-based gesture control interface," the 5th IEEE Conference on Industrial Electronics and Applications, pp. 1522-1525, June 2010.

(9) Xing Su, Hanghang Tong, and Ping Ji, "Activity recognition with smartphone sensors," vol. 19, no. 3, Tsinghua Science and Technology, pp. 235 - 249, June 2014.

(10) J. L. Reyes-Ortiz, "Smartphone-based human activity recognition," Springer Publishing Company, 2015.

(11)D. Anguita et. al., "A public domain dataset for human activity recognition using smartphones,"21st European Symposium on Artificial Neural Networks, Computational Intelligence and Machine Learning, Bruges, Belgium, April 24-26, 2013.

(12) J. C. Quiroz et. al., "Feature selection for activity recognition from smartphone accelerometer data," Journal of Intelligent Automation \& Soft Computing, pp. 1-9, July 2017.

(13)F. J. Ordo'nez and D. Roggen, "Deep convolutional and LSTM recurrent neural networks for multimodal wearable activity recognition," Sensors, vol. 16, no. 115, pp.1-25, 2016.

(14) N. Y. Hammerla, S. Halloran, and T. Ploetz, "Deep, convolutional, and recurrent models for human activity recognition using wearables," arXiv:1604.08880, 2016. 